\title{
Content Centric Services in Smart Cities
}

\author{
I. Cianci, G. Piro, L. A. Grieco, G. Boggia, and P. Camarda \\ DEE - Dip. di Elettrotecnica ed Elettronica, Politecnico di Bari \\ v. Orabona, 4 - 70125, Bari, Italy. \\ Email: \{i.cianci,g.piro,a.grieco,g.boggia,camarda\}@poliba.it
}

\begin{abstract}
We expect around 9 billions people on the Earth within $2050,70 \%$ of them living in urban environments. By that time, we have to be ready to support a never seen request for ICT services in order to improve the efficiency of future Smart Cities, thus allowing a pacific coexistence among humans. In line with this premise, the present contribution proposes a novel service platform, based on the emerging content-centric networking paradigm. It can easily embrace all available, upcoming, and remote generation wireless techologies, while supporting, at the same time, ubiquitous and secure applications in many domains, such as: e-helthcare, intelligent transportation systems, entertainment, and many others. All details of the approach we propose, have been carefully described by means of pragmatical use-cases, thus making evident its effectivenes in future realistic urban environments.
\end{abstract}

\section{INTRODUCTION}

The Smart City vision is born as a response to the challenges arising from the steadily growing number of people living in urban agglomerations [1]. While its exact definition has not been formulated yet, a Smart City should provide a high quality of life to all its citizens, mainly following six driver innovation axes [2]: smart mobility, smart environment, smart people, smart living, and, finally, smart governance. The main goal is to create a business-competitive and attractive environment by leveraging on the human capital of the city, while, at the same time, allowing a wide participation to the public life and developing novel ways to look at transportation systems and natural resource preservation themes.

Information \& Communication Technology (ICT) infrastructures are at the basis of the smart city foundation, because they are strictly required to provide advanced services in Intelligent Transport System (ITS), environmental and energy monitoring, building management, healthcare, public safety and security, and remote working and e-commerce domains [3], [4]. In other words, they can play a key role to internetwork all the actors of the Smart City [5] and to support the provisioning of seamless ubiquitous services [6].

From a technological point of view, we are at a turning point where, from one side, there is a wide availability of wireless communication technologies, such as the Internet of Things (IoT) [7], the novel cellular architectures based on Long Term Evolution (LTE) and LTE Advanced (LTE-A) specifications [8], the Worldwide Interoperability for Microwave Access (WiMAX) [9], and the Wireless Access in Vehicular Environments (WAVE) protocol stack [10], while, from the other hand, we are observing a radical change in the way digital resources are used. Nowadays, in fact, users are interested to sharing contents rather than interconnecting themselves to remote devices [11]. As a consequence, to support mobile applications in Smart Cities, a change of perspective is required: ICT platforms, in fact, should be content-oriented and, at the same time, able to embrace the wide availability of cutting-edge wireless communication systems.

While some preliminary attempts in this direction have been done in [3], [4], all aforementioned issues remain still open, asking for novel, solid, and effective solutions.

In addition, many interesting proposals for the Future Internet are emerging to sustain the content-oriented demands of the next generation users [11]. Among them the ContentCentric Networking $(\mathrm{CCN})$ architecture, conceived at the Palo Alto Research Center, is considered as a very promising solution. In its rationale, contents become independent entities, including all the mechanisms useful to verify their integrity and validity; doing so, all users can retrieve contents without having any awareness about the location of service providers [12].

In this ebullient panorama of activities, we devise herein a $\mathrm{CCN}$-oriented service platform for Smart Cities, able to cope with the communication needs of future urban environments. Based on a content oriented approach, it can integrate all the most relevant wireless communication technologies, thus fulfilling three main objectives: providing ubiquitous services to citizens, optimizing the usage of communication resources thanks to distributed caching mechanisms, and solving security issues by leveraging on the peculiarities of the $\mathrm{CCN}$ architecture.

The rest of the paper is organized as follows: in Secs. II and III a brief review of the most appealing wireless technologies and of the CCN rationale are presented, respectively. Sec. IV describes the service platform we envisage in this contribution, providing practical examples and use cases of smart services it can enable. Finally, Sec. V draws conclusions and forecasts future research.

\section{ENABLING WIRELESS TECHNOLOGIES}

The aim of this Section is to briefly review the most promising wireless technologies for Smart Cities, including IoT protocol stacks, vehicular architectures, WiMAX, and next generation cellular systems.

\section{A. Internet of Things}

In IoT scenarios, a potentially (very) large number of nodes is able to establish low-power short-range wireless links, 
thus forming a capillary networking infrastructure that can be connected to the Internet [7]. Thanks to this paradigm, pervasive and Smart ICT services, pertaining to transportation and logistics, healthcare, smart environment (home, office, plant), and personal/social domains, can be furnished by providing real-life objects with IoT-enabled communication devices. To translate in reality the IoT vision, IEEE and IETF standardization bodies have recently proposed several interesting protocols that, if properly integrated, can ease plug and play operations of smart devices in IPv6 networks. In brief, they include:

- IEEE 802.15.4 MAC [9]: it is a low-power MAC/PHY protocol for enabling short range communications in Personal Area Networks (PAN). Its efficiency can be greatly boosted with the IEEE 802.15.4e amendment ${ }^{1}$, which, exploiting Time Slotted Channel Hopping (TSCH), enhances throughput, reliability, and energy efficiency of wireless PAN [13].

- 6LoWPAN [7]: IPv6 over Low-Power Wireless Personal Area Networks (6LoWPANs) has been mainly conceived as an adaptation layer to let IPv6 datagram to fit the small payload size (up to 127 bytes in IEEE 802.15.4) by means of advanced header compression techniques.

- RPL [7]: IPv6 Routing Protocol for Low power and Lossy Networks (RPL) is a gradient based routing protocol that can ease the formation and the management of multihop topologies based on short-range low-power links. It supports multiple roots and is highly flexible thanks to the possibility to optimize the topology based on parametric optimization functions.

- CoAP [14]: The IETF Constrained RESTful Environments (CORE) working group has defined the Constrained Application Protocol (CoAP) which easily translates to HTTP for integration with the web, while meeting specialized requirements such as: multicast support, very low overhead, and simplicity for constrained environments.

\section{B. Vehicular Ad-Hoc Networks}

Vehicular Ad-Hoc Networkss (VANETs) represent an emerging wireless technology allowing efficient communication among vehicles (i.e., cars, bus, and truck) and fixed devices positioned along the street [15]. MAC and PHY layers of such technology have been conceived by the IEEE 802.11p working group as an extension of the common IEEE 802.11 [9] standard. Moreover, architectures, applications, and protocols, as well as all management activities have been described into the the IEEE 1609 Family of Standards for WAVE [10] where the IEEE $802.11 \mathrm{p}$ specification is perfectly integrated.

VANET services can be classified into three different groups [15]:

- Safety: they have been conceived for reducing the probability to have road accidents and for avoiding vehicles

${ }^{1}$ IEEE 802.15.4e amendment has been released on April 2012. pile-up, resulting from accidents (or congestions) along the roads.

- Traffic control: they should improve the travel quality through the prevention of problems on the road (such as traffic jam and congestion).

- User applications: have been introduced in order to provide entertainment services (i.e., music and video sharing, chat, games, and Internet connectivity) to passengers in a vehicle.

Their adoption in Smart Cities would greatly ease the construction of a digital ecosystem that, surrounding vehicles, could enable intelligent transportation services for future citizens.

\section{Worldwide Interoperability for Microwave Access}

The IEEE 802.16 standard [9], widely known as WiMAX, defines the MAC and PHY layers specifications for broadband wireless access networks. WiMAX offers an alternative to wired networks, such as coaxial systems using cable modems, fiber optics and DSL (Digital Subscriber Line). It provides high speed data access to large geographical areas, covering distance up to $50 \mathrm{~km}$ and supporting data rates up to $100 \mathrm{Mbps}$, depending on the distance from the base station and the underlying PHY layer. With this range and data rates, WiMAX can be adopted as a backhaul connection for enterprise campuses, WiFi hotspots and cellular networks.

A WiMAX network includes the base station (BS) and the associated subscribers stations and mobile subscribers.

The MAC layer of WiMAX supports Point-to-Multipoint (PMP) and Mesh topologies. In PMP topology, the BS serves a number of stations that are in the same broadcast range, like a cellular structure. In mesh topologies, instead, ad-hoc environments can be set-up.

The deployment of WiMAX would be very useful to improve the wireless connectivity in those areas that are not well covered by cellular systems. In this way, it would be possible to reduce the digital divide and to provide broadband access to a wider share of people living in urban environments.

\section{Long Term Evolution and LTE-A}

The requirements of LTE networks are very ambitious [8]: they will provide high peak data rates (up to $100 \mathrm{Mbps}$ in downlink and $50 \mathrm{Mbps}$ in uplink with $20 \mathrm{Mhz}$ of bandwidth), increased cell edge throughput, less than $5 \mathrm{~ms}$ user plane latency, significant reduction of control plane latency, support for high user mobility, scalable bandwidth from 1.25 to 20 $\mathrm{MHz}$, and enhanced support for end-to-end QoS. To fulfill these goals, the Radio Resource Management block has been designed to support a mix of advanced MAC and Physical functionalities, like packet scheduling, link adaptation, and Hybrid ARQ. The LTE technology will replace current 3G systems to provide a higher spectral efficiency and to support the ever growing bandwidth demands of mobile applications in urban environments. Thus, it can be viewed as the basic underlying wireless infrastructure of future smart cities. 
In order to further increase the capacity and the coverage of mobile communication systems, the 3GPP has conceived within the LTE-A system (i.e., the natural evolution of LTE) innovative technological solutions, such as carrier aggregation, enhanced multi-antenna support, Coordinated Multi-Point transmission techniques, relaying, multi-user multiple input multiple output communications, thus allowing such kind of wireless networks to work very close to the Shannon limit.

In addition, the idea to deploy a Heterogeneous Network (HetNet) composed by macro and small-range (i.e., micro, pico, and femto) cells as been also introduced. Micro and pico cells could be exploited for enhancing coverage and capacity in some regions inside the macro one. Whereas, femtocells have been devised for offering broadband services in indoor (home and offices) and outdoor scenarios with a very limited geographical coverage [8].

Without any doubt, the adoption of the HetNet paradigm would foster the diffusion of next generation broadband networks, thus providing a valid support for all the innovative services devised for Smart Cities.

To complete our discussion, we reported in Tab. I some details about aforementioned wireless technologies, highlighting, for each of them, main target services they have been originally designed for.

\section{CCN IN A NUTSHELL}

During last years, content-sharing Internet applications have become dominant, thus making evident the main limitations of the current IP architecture, which is born, instead, to support connection-less packet exchange among remote hosts [11].

The so-called Future Internet represents a novel way to look at planetary packet-based communication systems that should account for the emerging usage models of digital resources, thus solving some of the current Internet problems (e.g., efficiency, scalability, mobility management) and fullfilling users' requirements (i.e., more efficient, secure, and integrated media experience) [11].

At the present stage, many valid Future Internet architectures have been proposed. Some of them, like the Publish Subscribe Internet Routing Paradigm, the 4WARD NetInf project, and the Cache-and-Forward Network Architecture foster a radical change of the existing protocols [16]. Other ones, instead, such as the Data-Oriented Network Architecture [16] and the CCN approach [12], can be deployed by means of an "overlay" network (i.e., a logical network built on top of one or more existing IP networks).

$\mathrm{CCN}$ is part of the project Named Data Networking, and it is based on a "data-centric" approach, that makes unnecessary the mapping from what the users care about to the network's where. According to this new paradigm, all contents should be identified by a unique name, allowing users to retrieve information without having any awareness about the physical location of servers (e.g., IP address). Furthermore, CCN targets receiver-driven communications, based on content chunks exchange, name-based routing, and self-certifying packets [12].
We focus on $\mathrm{CCN}$ because it can greatly improve network performance by solving many problems of the existing protocols, it is fully compliant with existing infrastructures (hardware and protocols), and it enables secure data sharing applications. Further, it brings to a reduction of network congestion, data retrieval time, and computational load of servers.

Main CCN features can be summarized in (i) chunks segmentation of contents, (ii) introduction of strategy and security layers to face message dissemination and security issues, respectively, and (iii) IP as the underlying technology on top of which the CCN overlay is deployed (as shown in Fig. 1). Nevertheless, there are several open issues in the big topic of CCNs, such as: (i) routing, (ii) congestion control, (iii) strategy layer design, (iv) name space design, (v) semantic layer and (vi) accurate models.

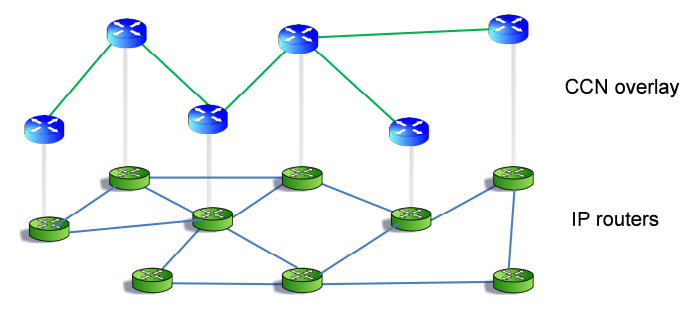

Fig. 1. IP network and CCN overlay

As specified before, CCN communications are driven by the consumer of data and only two types of messages are exchanged (namely Interest and Data). A user may ask for a content by issuing an Interest, which is routed within the overlay towards the nodes in posses of the required information, thus triggering them to reply with Data packets.

The routing operations are performed by the strategy layer only for Interest packets. Data messages, instead, just follow the reverse path to the requesting user, allowing every intermediate node to cache the forwarded content. Main fields of both Interest and Data packets ${ }^{2}$ are reported in Tab. II.

$\mathrm{CCN}$ adopts a hierarchical structure for names, which leads to a name tree. In particular, it is formed by several components, each one made by a number of arbitrary octets (optionally encrypted), so that every name prefix identifies a sub-tree in the name space. An Interest can specify the full name of the content or its prefix, thus accessing to the entire collection of elements under that prefix.

Finally, since contents are exchanged based on their names, multiple nodes interested to a particular data can share it using multicast suppression techniques over a broadcast medium [17].

\section{The ENVISAged SERVICE PLATFORM}

\section{A. Why do we need for a CCN-oriented service platform ?}

In future urban contexts, people will be surrounded by a ubiquitous digital eco-system made by internet-connected vehicles, smart buildings, and a myriad of other equipments

\footnotetext{
${ }^{2}$ The considered packet structures is the one proposed within the $\mathrm{CCNx}$ implementation, available at www.ccnx.org
} 
TABLE I

ADDITIONAL INFORMATION ON CONSIDERED WIRELESS TECHNOLOGIES

\begin{tabular}{|c|c|c|c|c|}
\hline Technology & Spectrum & Capacity & Coverage & Main services \\
\hline IoT & $\begin{array}{l}16 \text { channels of } 5 \mathrm{MHz} \\
\text { each at } 2.4 \mathrm{GHz}\end{array}$ & $\leq 0.25 \mathrm{Mbps}$ & $\leq 10 \mathrm{~m}$ & $\begin{array}{l}\text { sensing, data collection, short-range interac- } \\
\text { tions, machine-to-machine communications }\end{array}$ \\
\hline VANET & $10 \mathrm{Mhz}$ at $5.9 \mathrm{GHz}$ & $\leq 54 \mathrm{Mbps}$ & $\leq 500 \mathrm{~m}$ & safety, traffic control, and user applications \\
\hline WiMAX & $20 \mathrm{MHz}$ at $5 \mathrm{GHz}$ & $\overline{\leq} 100 \mathrm{Mbps}$ & $\begin{array}{l}\text { up to } 50 \mathrm{~km} \text {, optimized in } \\
\text { the range }[1.5-5] \mathrm{km}\end{array}$ & broadband services \\
\hline LTE & $\begin{array}{l}\text { from } 1.4 \text { to } 20 \mathrm{MHz} \text { at } 2 \\
\mathrm{GHz}\end{array}$ & $\begin{array}{l}\leq 100 \text { Mbps in downlink; } \\
\leq 50 \text { MBps in uplink }\end{array}$ & $\begin{array}{l}\text { up to } 100 \mathrm{~km} \text { with a slight } \\
\text { degradation after } 30 \mathrm{~km}\end{array}$ & $\begin{array}{l}\text { voice, video streaming, web applications, } \\
\text { broadband services }\end{array}$ \\
\hline LTE-A & up to $100 \mathrm{MHz}$ at $2 \mathrm{GHz}$ & $\leq 1 \mathrm{Gbps}$ & $\begin{array}{l}\text { up to } 100 \mathrm{~km} \text { with a slight } \\
\text { degradation after } 30 \mathrm{~km}\end{array}$ & $\begin{array}{l}\text { voice, video streaming, web applications, } \\
\text { broadband services }\end{array}$ \\
\hline
\end{tabular}

TABLE II

MAIN FIELDS OF CCN MESSAGES

\begin{tabular}{|c|c|c|}
\hline Packet & Field & Description \\
\hline \multirow[t]{3}{*}{ 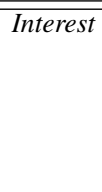 } & $\begin{array}{l}\text { Content } \\
\text { Name }\end{array}$ & $\begin{array}{l}\text { Identifies the requested item. It is formed by several } \\
\text { components that identify a sub-tree in the name } \\
\text { space. }\end{array}$ \\
\hline & nonce & Suppresses possible duplicates. \\
\hline & $\begin{array}{l}\text { Publisher } \\
\text { Filter }\end{array}$ & $\begin{array}{l}\text { Imposes that only a specific user can answer to that } \\
\text { Interest. }\end{array}$ \\
\hline \multirow[t]{5}{*}{ Data } & $\begin{array}{l}\text { Content } \\
\text { Name }\end{array}$ & Identifies the requested item. \\
\hline & $\begin{array}{l}\text { Publisher } \\
\text { ID }\end{array}$ & Identifies the users that has generated the data. \\
\hline & Signature & Guarantees the publisher authentication. \\
\hline & $\begin{array}{l}\text { Stale } \\
\text { Time }\end{array}$ & $\begin{array}{l}\text { Defines the life time of the carried payload. It is } \\
\text { used for scheduling caching operations (eventually } \\
\text { prohibited). }\end{array}$ \\
\hline & Data & It is the content with an arbitrary length. \\
\hline
\end{tabular}

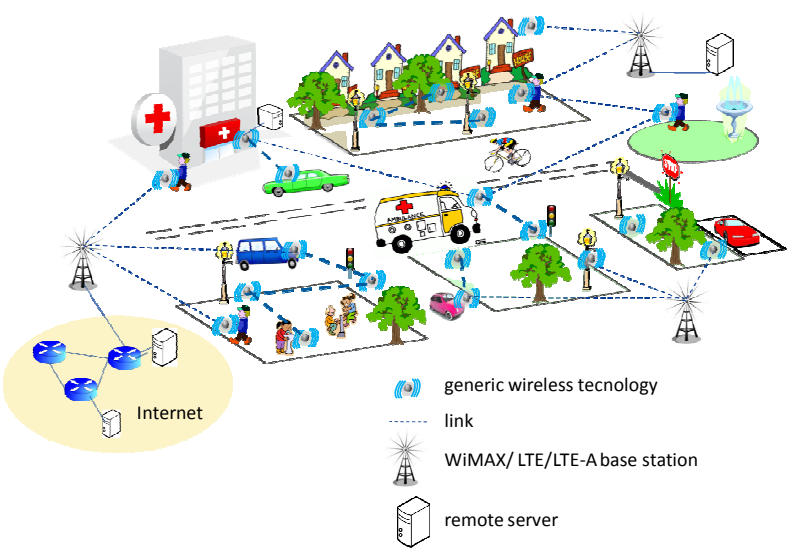

Fig. 2. A smart city

(such as computer, tablet, smartphone, GPS navigation devices, sensors, etc.) willing to interact to each other. To provide a clear example, Fig. 2 envisions a possible upcoming scenario where pedestrians will be equipped with their LTE/LTE-A smartphones and share multimedia contents, while vehicles exchange traffic information, and sensors measure and deliver specific environmental variables by exploiting machine-tomachine communication technologies. Going beyond visions, the provisioning of ubiquitous services in a Smart City will be likely to be influenced by the fast evolving panorama of wireless technologies, which are going to appear on the market, as well as by novel data-centric ways to look at user applications. Therefore, future ICT platforms are expected to be able to face:

- multi-homing: a device with one o more network interfaces,i.e., multi-homed, should be able to access to Smart City services regardless of the communication system it adopts;

- service heterogeneity: a huge number of services has to be supported, such as IoT, e-healthcare, Smart Transportation and ITS, Entertainment, E-commerce, and many others, which have very different requirements;

- efficiency: ICT resources have to be handled in a efficient way, thus guaranteeing low energy consumptions along with the expected level of Quality of Experience (QoE);

- security and mobility: they have to be natively supported;

- content-oriented network usage: the compatibility with emerging Future Internet architectures has to be ensured because the way users look at digital resources is very quickly approaching a content-oriented model.

The content centric paradigm can be a valid answer to all these issues. It promotes, in fact, an overlay approach that, from one side, can ease the design of universal and secure Smart City applications, whereas, from the other hand, can better support a content-oriented approach to network services, improve the efficiency of communication systems, and seamlessly integrate mobile technologies.

\section{B. CCN-oriented service platform: design principles}

Fig. 3 pictures the $\mathrm{CCN}$-oriented platform we propose: it is built on two levels, i.e., the service layer and the technology layer, which interact among them through the $\mathrm{CCN}$ interface. It is important to underline that the design of the name tree is a key aspect, because the Content Name field influences the way Interest and Data packets are treated. Starting from the root tree, identified with "/", there will be a different sub-tree for each offered service and each one can have other sub-subtrees and so on, i.e. "/service/sub - category/sub-subcategory/additional - info/". We note that an optimized name tree could give enormous advantages for routing operations and packet processing.

Since CCN has been conceived to operate on top of unreliable packets delivery services, UDP can be used at the 


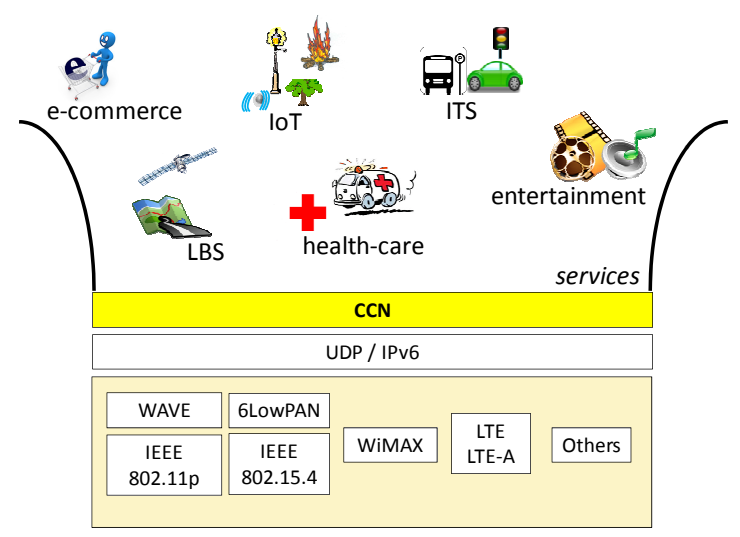

Fig. 3. The CCN-oriented service platform

transport layer.

Furthermore, a very important problem to face is related to the procedure each user should follow to joins the overlay network and participate to a given set of services. A possible solution is the adoption of IPv6 that, besides many other advantages it can bring, such as expanded address space, self-configuring capabilities, and stronger support to mobility and security, could provide a very beneficial contribution by mapping each service family on a different multicast group through Multicast Listener Discovery (MLD) signaling [18].

Otherwise, each device could be identified by an unique IPv4/IPv6 address and it can offer a particular service by diffusing prefix announcements, by means of mechanisms already supported by $\mathrm{CCN}$.

Next sub-sections will describe, using example use-cases, the main details of the architecture we are proposing.

\section{Use Cases}

1) Health-care service: Now, we consider the example of an LTE user that wants to book an orthopedic examination in a public hospital of his town. First of all, the CCN daemon running on top of his smartphone has to join the multicast group "Healthcare". The second operation is the discovery of the public hospitals in the town, performed by diffusing an Interest packet with name /healthcare/discovery/. Each healthcare service provider will answer with a Data packet. Thus, the considered user can choose the preferred hospital, for example HospitalX, and send a new Interest packet with parameters: /healthcare/reservation/encryptedName as name, HospitalX as Publisher Filter and other information such as orthopedic clinic, doctor, dates in the Info field. The hospital will answer with a Data packet with same name and the list of available data/time as encrypted payload. Then, the user will generate another Interest packet wherewith he accept a date and at the end, a Data packet with the receipt of successful reservation will be sent by the Hospital. These operations are summarized in Fig. 4.

It is important to note that in this specific example there is a first phase, in which other nodes can collaborate to the discovery process of the medical facilities (if they already have

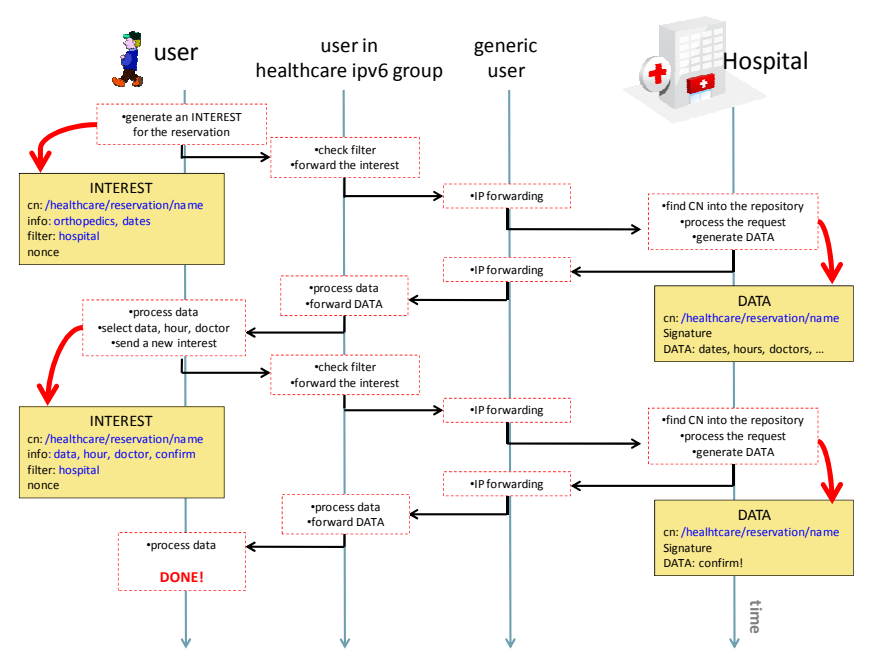

Fig. 4. Use case 1: an health-care service

that information in their caches). Then, the reservation cannot be handled using already cached contents but the $\mathrm{CCN}$ remains still useful for addressing reliability and security issues.

2) Entertainment service: Another type of service that a Smart City should offer falls in the Entertainment category. In fact, Cisco has estimated that by 2015 the most part of IP traffic will be related to multimedia traffic [19].

An example of an entertainment service in a Smart City, handled with the proposed architecture, is showed in Fig. 5. The considered scenario is composed by three users: the former posses a device with only the WAVE interface, the latter has a smarthphone with the LTE connectivity, and the middle one is equipped with a device having both of these technologies available. User-1 and User-2 are interested in $\operatorname{song} B$, which has been published by User-3. The CCN daemons, running on top of these devices, will join in the IPv6 multicast group Entertainment and will diffuse Interest packets with Content Name /entertainment/song/songB. Hence, each $\mathrm{CCN}$ node within such a group will receive the Interest and, in case it finds the required item in its cache, it will replay with a Data packets; otherwise, it will just forward the request to other nodes. In the interim, other nodes, which are not in the Entertainment service group, just will execute routing operations at the IP level. In this way, very popular contents will be found available in several caches at few hops, thus improving response times and lowering network congestions.

3) ITS service: The last use case refers to an ITS service. Transport is one of the pillars of modern society and ITS integrates different technological components in order to offer solution of general transport control and transport information services provision [20].

Focusing the attention on a VANET composed by a number of vehicles, each one equipped with a GPS device, we suppose that one of them, which is reaching an intersection, has to choose which street to take, i.e., streetA or streetB (see Fig. $6)$.

As starting point we assume that each node enjoying the ITS 


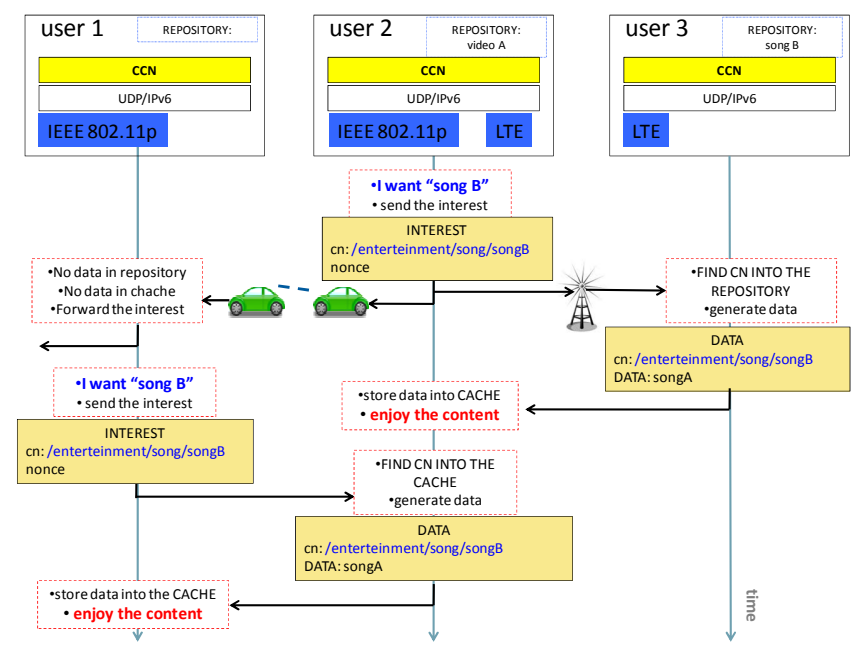

Fig. 5. Use case 2: an entertainment service

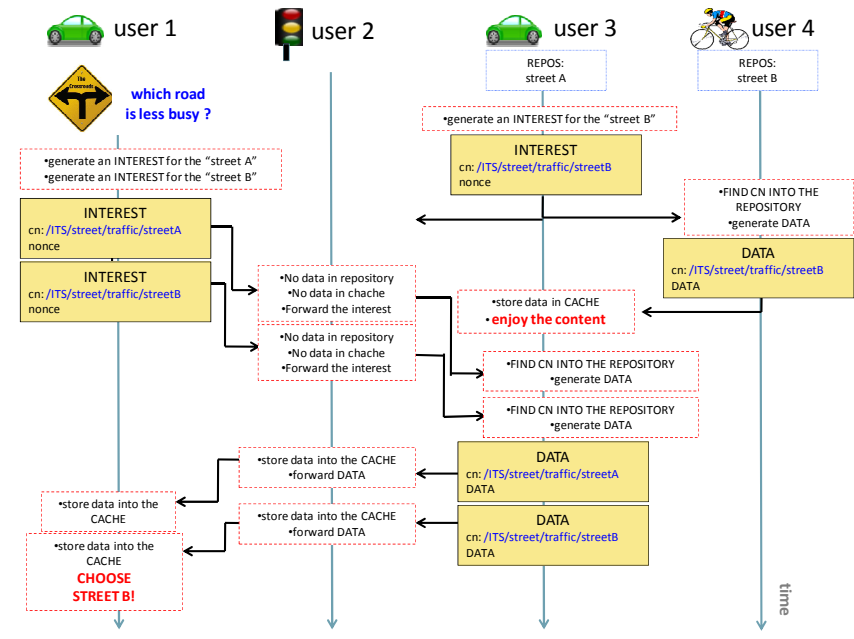

Fig. 6. Use case 3: an ITS service

service has in its cache the information related to the street it is in (such as average walking time, any work in progress, accidents, etc.). Hence, User-1 will generate two Interest asking for ITS/streets/traffic/streetA and ITS/streets/traffic/streetB. Each node receiving the Interest related to the street it has in cache, will replay with a Data packet containing all the requested information. Thus, all nodes among the reverse path to User-1 can cache additional information. In this way, the information exchange, including emergency alert, becomes faster and more efficient.

\section{Conclusions}

How to cope with ICT services in Smart Cities? This paper has proposed a possible approach to the problem by highlighting its relevance in real-life use cases, taken from eheatlcare, ITS, and entertainment application domains. At its foundation the $\mathrm{CCN}$ rationale has been adopted to improve the efficiency of communication networks, to ease mobility, to support security, and to exploit all the potentials of next generation wireless technologies and IoT. Future works will further explore this novel architecture to better discover the benefits that could derive from its wide deployment and to finely scavenge possible drawbacks. Moreover, its integration with specific web-oriented languages (such as those developed by the HL7 organization for heal-care services) will be also investigated.

\section{ACKNOLEDGMENTS}

This work was partially funded by the Apulia Regions Projects PS 025 (ICT supporting logistic services: a model of organized market) and the National Operative Program ERMES (Enhance Risk Management through Extended Sensors).

\section{REFERENCES}

[1] A. Caragliu, C. del Bo, and P. Nijkamp, "Smart cities in europe," in Proc. of 3rd Central European Conf. in Regional Science (CERS), Oct. 2009.

[2] R. Giffinger, "Smart cities - ranking of european medium-sized cities," Tech. Rep. Centre of Regional Science, Vienna UT, Oct. 2007.

[3] J. M. Hernndez-Muoz, J. B. Vercher, L. Muoz, J. A. Galache, M. Presser, L. A. H. Gmez, and J. Pettersson, "The future internet." SpringerVerlag, 2011, ch. Smart cities at the forefront of the future internet.

[4] H. Schaffers, N. Komninos, M. Pallot, B. Trousse, M. Nilsson, and A. Oliveira, "The future internet." Springer-Verlag, 2011, ch. Smart cities and the future internet: towards cooperation frameworks for open innovation.

[5] L. Anthopoulos and P. Fitsilis, "From digital to ubiquitous cities: Defining a common architecture for urban development," in Proc. of the IEEE Int. Conf. on Intelligent Environments (IE), Jul. 2010.

[6] O. Kwon and J. Kim, "A Methodology of Identifying Ubiquitous Smart Services for U-City Development," in Proc. of the Int. Conf. on Ubiquitous Intelligence and Computing (UIC), Jul. 2007.

[7] O. Hersent, D. Boswarthick, and O. Elloumi, The Internet of Things: Key Applications and Protocols, 2nd ed. Wiley, 2012.

[8] E. Dahlman, S. Parkvall, and J. Skld, 4G LTE/LTE-Advanced for Mobile Broadband. Academic Press, 2011.

[9] B. H. Walke, S. Mangold, and L. Berlemann, IEEE 802 Wireless Systems. NJ, USA: John Wiley \& Sons, Ltd, 2006.

[10] IEEE, Wireless Access in Vehicular Environments (WAVE): IEEE 1609 Standards Series, IEEE Std. 1609, 2009.

[11] B. Ahlgren, P. A. Aranda, P. Chemouil, S. Oueslati, L. M. Correia, H. Karl, M. Sollner, and A. Welin, "Content, connectivity, and cloud: ingredients for the network of the future," IEEE Commun. Mag., vol. 49, no. 7, Jul. 2011.

[12] V. Jacobson, D. K. Smetters, J. D. Thornton, M. F. Plass, N. H. Briggs, and R. L. Braynard, "Networking named content," in Proc. of Conf. Next (CONEXT), Dec. 2009.

[13] A. Bachir, M. Dohler, T. Watteyne, and K. Leung, "MAC Essentials for Wireless Sensor Networks," IEEE Commun. Surv. Tuts, vol. 12, no. 2, Apr. 2010.

[14] Z. Shelby, K. Hartke, C. Bormann, and B. Frank, Constrained Application Protocol (CoAP) - Internet-Draft, IETF CoRE Working Group, Mar. 2012.

[15] Y. Toor, P. Muhlethaler, and A. Laouiti, "Vehicle ad hoc networks: applications and related technical issues," IEEE Commun. Surv. Tuts, vol. 10, no. 3, Sep. 2008.

[16] B. Ahlgren, C. Dannewitz, C. Imbrenda, D. Kutscher, and B. Ohlman, "A Survey of Information-Centric Networking (Draft)," in InformationCentric Networking. Schloss Dagstuhl, 2010.

[17] B. Adamson, C. Bormann, M. Handley, and J. Macker, "RFC 5401 Multicast Negative-Acknowledgment (NACK) Building Blocks," IETF, Nov. 2008.

[18] P. Loshin, IPv6: Theory, Protocol, and Practice, 2nd ed. Morgan Kaufmann, 2004

[19] "Cisco visual networking index: Forecast and methodology, 2010-2015," White Paper, Cisco, Jun. 2011.

[20] B. Drilo, D. Saric, and R. Filjar, "The role of telecommunications in development of new-generation intelligent transport systems," in International Conference on Wireless VITAE, May 2009. 